\title{
Les spectromètres à haute résolution
}

\author{
M. Johnson, J. Combet et B. Frick
}

Institut Laue-Langevin, BP. 156, 38042 Grenoble, France

\begin{abstract}
Résumé : Cette article présente les spectrometres à haute résolution de type rétrodiffusion. Un rappel des principes fondamentaux est suivi par une description du parc d'instruments actuels. L'utilité de la technique est démontrée à travers plusieurs exemples d'experiences et une présentation du traitement des données souligne les problèmes associés à ce type d'expérience. Finalement, la technique de rétrodiffusion est en cours d'êvolution et les projets d'instruments les plus significatifs sont présentés.
\end{abstract}

\section{PRINCIPES DE LA TECHNIQUE DE RETRODIFFUSION}

La longueur d'onde $\lambda$ réfléchie par un cristal est donnée par la loi de Bragg $n \lambda=2 d \sin \theta$, où $d$ est l'espacement entre les plans atomiques, $\theta$ l'angle entre ces plans et le faisceau incident et $n$ un entier. Pour une valeur fixée de $d$, elle varie en fonction de l'angle $\theta$. La largeur spectrale $(\delta \lambda)$ du faisceau réfléchi est donnée par la différentielle de cette équation, $\delta \lambda \lambda=\delta d / d+(\cos \theta / \sin \theta) \delta \theta$. Elle présente un minimum lorsque $\theta$ est proche de $90^{\circ}$, c'est à dire, lorsque le faisceau est réfléchi sur lui-même $\left(2 \theta=180^{\circ}\right)$ ou rétrodiffusé (voir fig. 1). La résolution spectrale est donc optimisée lorsque le faisceau de neutrons est rétrodiffusé. C'est Maier-Leibnitz [1], en 1966, qui a proposé pour la première fois d'utiliser la rétrodiffusion $(\mathrm{RD})$ des neutrons pour réaliser des études spectroscopiques à très haute résolution, bien que Alefeld figure seul sur la première publication [2].

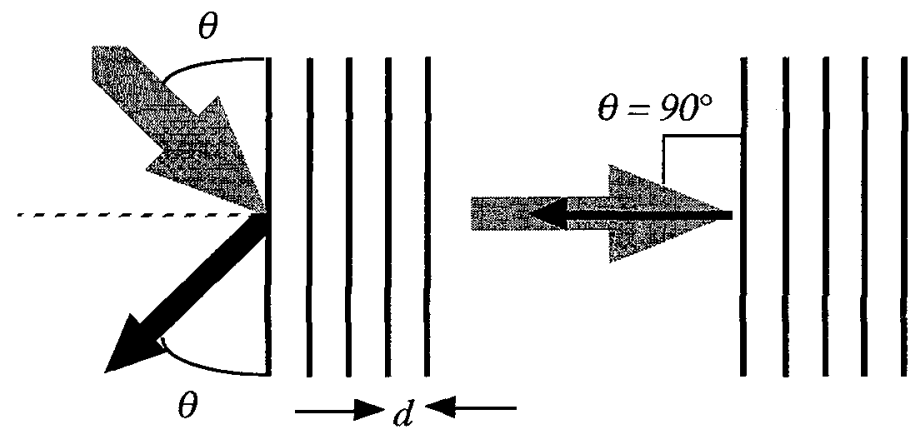

Figure 1 : Principe de la rétrodiffusion d'un faisceau de neutrons. La largeur des flèches correspond à $\delta \lambda$.

Dans le cas d'une géometrie proche de $\theta=90^{\circ}$ la largeur spectrale est donnée par [3]:

$\delta \lambda / \lambda=\delta \tau / \tau+\delta \alpha^{2} / 4$, où $\tau(\tau=2 \pi / d)$ est le vecteur de diffusion et $\alpha$ est la demi-divergence du faisceau (ou l'angle de déviation à la rétrodiffusion). Il existe en réalité une valeur limite pour $\delta \tau / \tau$ qui dépend du type de cristal et qui est donnée par "l'extinction primaire" ou la "largeur de Darwin" [4]. Pour les cristaux de silicium, la "largeur de Darwin" limite la résolution spectrale à $0.16 \mu \mathrm{eV}$ (FWHM). Expérimentalement, une largeur de $0.17 \mu \mathrm{eV}$ (FWHM) a été mesurée sur IN 16 dans une configuration très particulière minimisant la 
contribution $\delta \alpha$ [5]. Pour la plupart des spectromètres à $R D$, c'est en fait la divergence du faisceau, ou la déviation à la rétrodiffusion qui domine la résolution spectrale.

Sur les spectromètres classiques, le principe de rétrodiffusion est utilisé à diverses étapes pour optimiser la résolution de l'instrument. Dans le spectromètre primaire tout d'abord, où le faisceau est mis en forme par une réflexion normale au cristal monochromateur. Dans le spectromètre secondaire ensuite, où l'énergie finale des neutrons diffusés par l'echantillon est contrôlée par une réflexion normale aux analyseurs.

La résolution de l'instrument détermine la limite inférieure du transfert d'énergie observable $(\sim 0.3 \mu \mathrm{eV})$, ainsi que la limite supérieure des temps de corrélation des mouvements observables (mouvement diffusifs, $\tau_{c}$ $<2$ ns). En terme de résolution, ou de temps de corrélation accessibles, la technique de la RD se situe ainsi entre les techniques de temps-de-vol (TdV) et d'écho de spins (EdS). Par TdV [6], la meilleure résolution est de l'ordre de $10 \mu \mathrm{eV}(\lambda=12 \AA)$. A une longueur d'onde (et à un transfert de moment) similaire à celle de la $\mathrm{RD}(\lambda=6 \AA)$, la résolution est proche de $60 \mu \mathrm{eV}$. Par EdS [7] sur IN11, le temps de Fourier (la fenêtre temporelle pour mesurer $\tau_{c}$ ) peut atteindre $50 \mathrm{~ns}$ à $\lambda=12 \AA$. Une fois de plus, à $\lambda=6 \AA$, la limite supérieure est de $10 \mathrm{~ns}$. Sur IN15 cette limite est 10 fois supérieure (100ns). Lors d'études expérimentales, les mesures de type RD sont souvent complétées par des mesures de TdV et de EdS afin de couvrir une plus large fenêtre dynamique.

Il existe également une limite supérieure du transfert d'énergie. Comme nous le verrons, celle-ci varie avec la méthode utilisée pour moduler l'énergie incidente. Dans la configuration classique, celle ci est modifiée par effet Doppler. La fenêtre en énergie est typiquement de $\pm 15 \mu \mathrm{eV}$. Grâce à des machines Doppler plus performantes, cette limite peut être repoussée à $\pm 40 \mu \mathrm{eV}$. Une alternative à l'effet Doppler consiste à modifier l'énergie incidente en modifiant la température du monochromateur. Par cette méthode il est possible d'élargir la fenêtre en énergie jusqu'à $200 \mu \mathrm{eV}$. Selon le cristal utilisé comme monochromateur, la fenêtre explorée peut inclure le pic élastique, ou au contraire être totalement inélastique. Cette deuxième configuration permet d'avoir une résolution de $\sim 1 \mu \mathrm{eV}$ à des transferts d'énergie proches du meV.

Il existe une complémentarité importante entre la technique de RD et la résonance magnétique nucléaire. Par relaxation spin-réseau, des temps de corrélation plus longs et plus courts sont accessibles (voir fig. 2) [8]. Par des mesures directes de densité spectrale, des transferts d'énergie plus faibles sont accessibles, la limite supérieure étant donnée par la fréquence de Larmor dans le champ magnétique le plus fort ( $5 \mathrm{~T})$ [9].

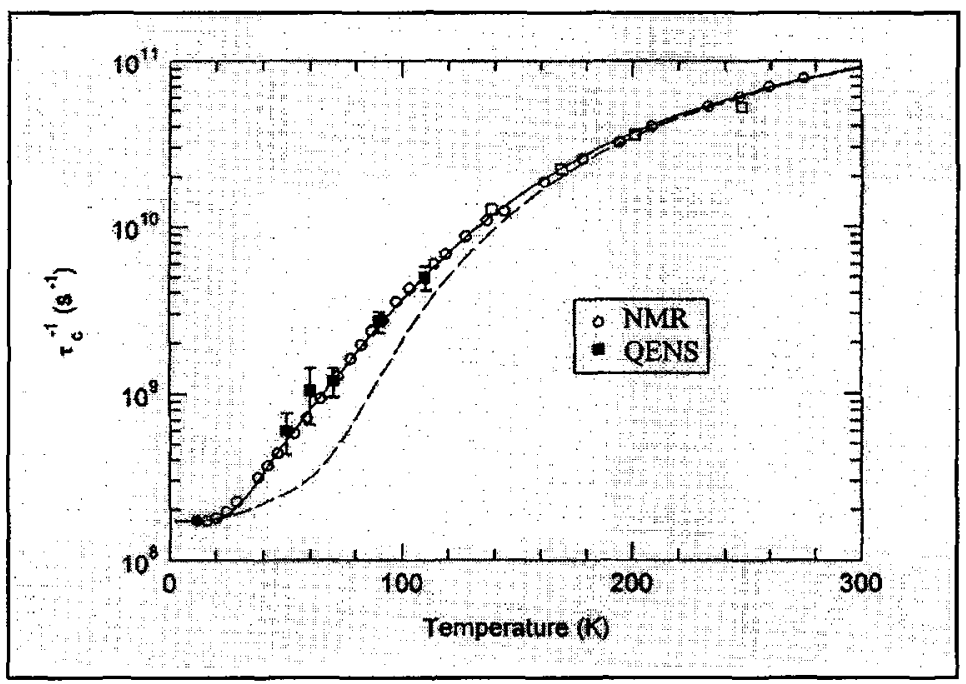

Figure 2 : Un exemple de la complémentarité entre la RD et la RMN : le temps de corrélations pour le transfert de proton dans l'acide benzoïque. 


\section{LES INSTRUMENTS DE RETRODIFFUSION}

La caractéristique principale de tous les spectromètres à $\mathrm{RD}$ est que l'énergie finale des neutrons est constante et dépend de la nature des cristaux analyseurs. Dans la plupart des cas les analyseurs sont formés de cristaux de silicium coupés dans le plan $(1,1,1)(d=3.135 \AA)$; l'énergie finale analysée étant de 2.080 $\operatorname{meV}\left(\lambda_{f}=6.271 \AA\right)$.

Afin d'obtenir un spectre en énergie il faut alors faire varier l'énergie incidente. Ceci est réalisé au niveau du spectromètre primaire. Seuls les neutrons ayant échangé une énergie telle que

Eincident $+\Delta \mathrm{E}=$ Eanalyseurs

sont détectés. La connaissance de Eincident et de Eanalyseurs permet de determiner la variation d'energie $\Delta \mathrm{E}$ liée à l'échantillon. Plusieurs méthodes sont couramment utilisées pour moduler l'énergie incidente. Sur la majeur partie des spectromètres, elle est obtenue par effet Doppler. Le cristal monochromateur est monté sur un piston et effectue un mouvement de translation. La vitesse maximum du monochromateur (quelques $\mathrm{m} / \mathrm{s}$ ) détermine alors le maximum de transfert d'énergie accessible. Quand le neutron est détecté, la vitesse du monochromateur, et ainsi l'énergie incidente, est déduite à partir du TdV correspondant au chemin entre le monochromateur et le détecteur (dans l'approximation où ce temps est indépendant du transfert d'énergie).

La variation de l'énergie incidente peut également être obtenue par modifications des paramètres de da maille du monochromateur $(d)$. Ceci est réalisé en chauffant ou en refroidissant le cristal monochromateur.

La validité de l'analyse TdV des neutrons dépend du fait que les neutrons détectés ont tous parcouru le chemin monochromateur-échantillon-analyseur-détecteur. Les neutrons qui arrivent directement dans les détecteurs (sans passer par les analyseurs), et pour lesquels l'analyse en énergie n'est pas réalisable doivent être rejetés. Ceci est téalisé à l'aide d'un chopper qui laisse passer des bouffés de neutrons. Au début de l'impulsion, les détecteurs sont inactifs pour une durée donnée par le TdV correspondant au chemin chopper-échantillon-analyseur-détecteur. Après quoi, le comptage commence jusqu'au début de la bouffé suivante. La fréquence de rotation du chopper est choisie de manière à ce que le faisceau incident soit stoppé vendant toute le durée du comptage.

Afin de respecter la géométrie de RD dans le spectromètre secondaire (voir fig.3), les détecteurs devraient être installés à la place de l'échantillon! Ceci n'étant pas réalisable, ils sont placés le plus près possible derrière l'échantillon. Les neutrons diffusés passent ainsi une deuxième fois à travers l'échantillon à leur retour des analyseurs. Plusieurs types de détecteurs peuvent utilisés. Selon leur géométrie, différentes configurations peuvent apparaître, chacune d'entre elle s'éloignant plus ou moins de la géométrie idéale de RD. Pour les détecteurs ponctuels (diamètre $4 \mathrm{~cm}$ ), la condition de RD doit être relâchée afín de focaliser l'image de chacun des analyseurs sur chaque détecteur. Ce problème peut être amélioré en utilisant un détecteur multitubes. Les analyseurs sont alors focalisés sur l'échantillon, tandis que leur image (renversée) est récupérée sur toute la hauteur des tubes. Dans tous les cas les détecteurs sont approximativement à $10 \mathrm{~cm}$ de l'échantillon.

Le premier spectromètre de type RD a été construit à Munich [10] et ses successeurs sont rapidement apparus à Jülich et à l'ILL. Actuellement il existe à l'LL trois spectromètres de type RD (IN10, IN13, IN16) chacun ayant des caractéristiques complémentaires. Ces trois spectromètres seront présentés cidessous. Un spectromètre BS similaire à IN10 existe également à Jülich. IRIS est le spectromètre à rétrodiffusion d'ISIS. Il sera également commenté car ces caractéristiques sont différentes, du fait qu'il est alimenté par une source de type spallation. Le parc des spectromètres à RD sera bientôt élargi avec l'arrivée de trois nouveaux appareils, encore plus performants, à NIST (en phase de tests) [11] à Munich, et à Los Alamos [12]. 


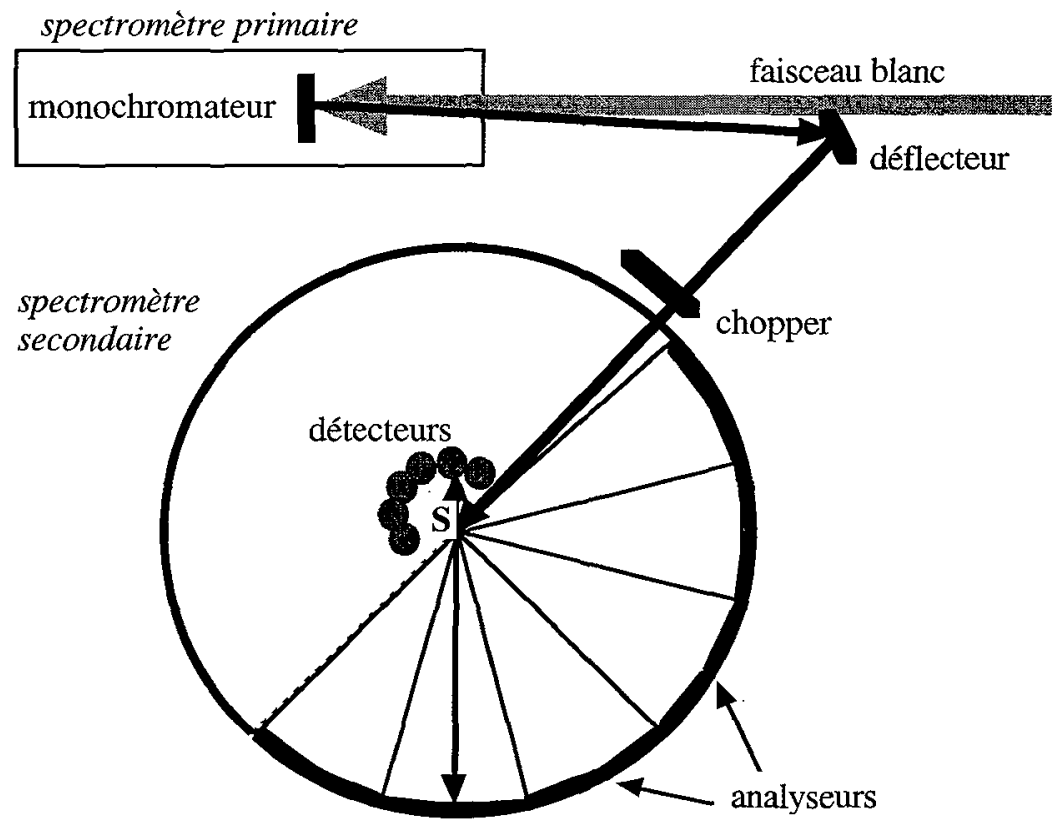

Figure 3 : Les éléments essentiels d'un spectromètre de type RD.

\subsection{IN10 $[13,14,15]$}

Ce spectromètre (fig.4), fonctionne depuis 1974. Il est le plus vieux de ce type de l'ILL. C'est pour cette raison qu'il fait preuve d'une grande versatilité. La configuration habituelle de l'instrument utilise un monochromateur et des analyseurs de silicium non polis, coupés selon le plan $(1,1,1)$. Les cristaux sont de taille assez importante (diamètre $10 \mathrm{~cm}$ ). Un seul cristal bicourbe (rayon \# 10m) est utilisé comme monochromateur $(8 \times 4 \mathrm{~cm})$. Au niveau du spectromètre secondaire les cristaux sont courbés (ou déformés) afin d'épouser la surface d'une sphère de rayon de $150 \mathrm{~cm}$. Les analyseurs ont une hauteur de $1 \mathrm{~m}$ et s'étendent sur une plage angulaire de $160^{\circ}$ afin de couvrir une angle solide important et d'optimiser le comptage. La fonction de résolution est Gaussienne de largeur à mi-hauteur $0.8 \mu \mathrm{eV}$. Le monochromateur est généralement monté sur une machine Doppler. Une fréquence maximale de $13.5 \mathrm{~Hz}$ permet d'atteindre des transferts d'énergie de $\pm 17 \mu \mathrm{eV}$. Il est possible d'obtenir une meilleure résolution en utilisant de petits cristaux polis (au niveau du monochromateur et des analyseurs). Ceci est lié au meilleur état de surface ainsi qu'à l'absence de déformation. Cela s'effectue cependant au détriment du flux car il existe beaucoup moins de neutrons satisfaisant la relation de Bragg (voir tableau I). Le flux et la résolution sont en effet étroitement liés aux variations de distances interéticulaires $\Delta d$. Le fait de courber les cristaux modifie ce paramètre introduit une distribution des distances. Il existe d'autant plus de neutrons satisfaisant la relation de Bragg, le flux est alors augmenté, tandis que la résolution s'élargit. Un autre inconvénient de la configuration "poli" est que la fonction de résolution a une forme Lorentzienne. En effet, l'amélioration de l'état de surface (et de la résolution) conduit à une courbe de réflexion comparable à celle de Darwin, qui présente des ailes importantes. 


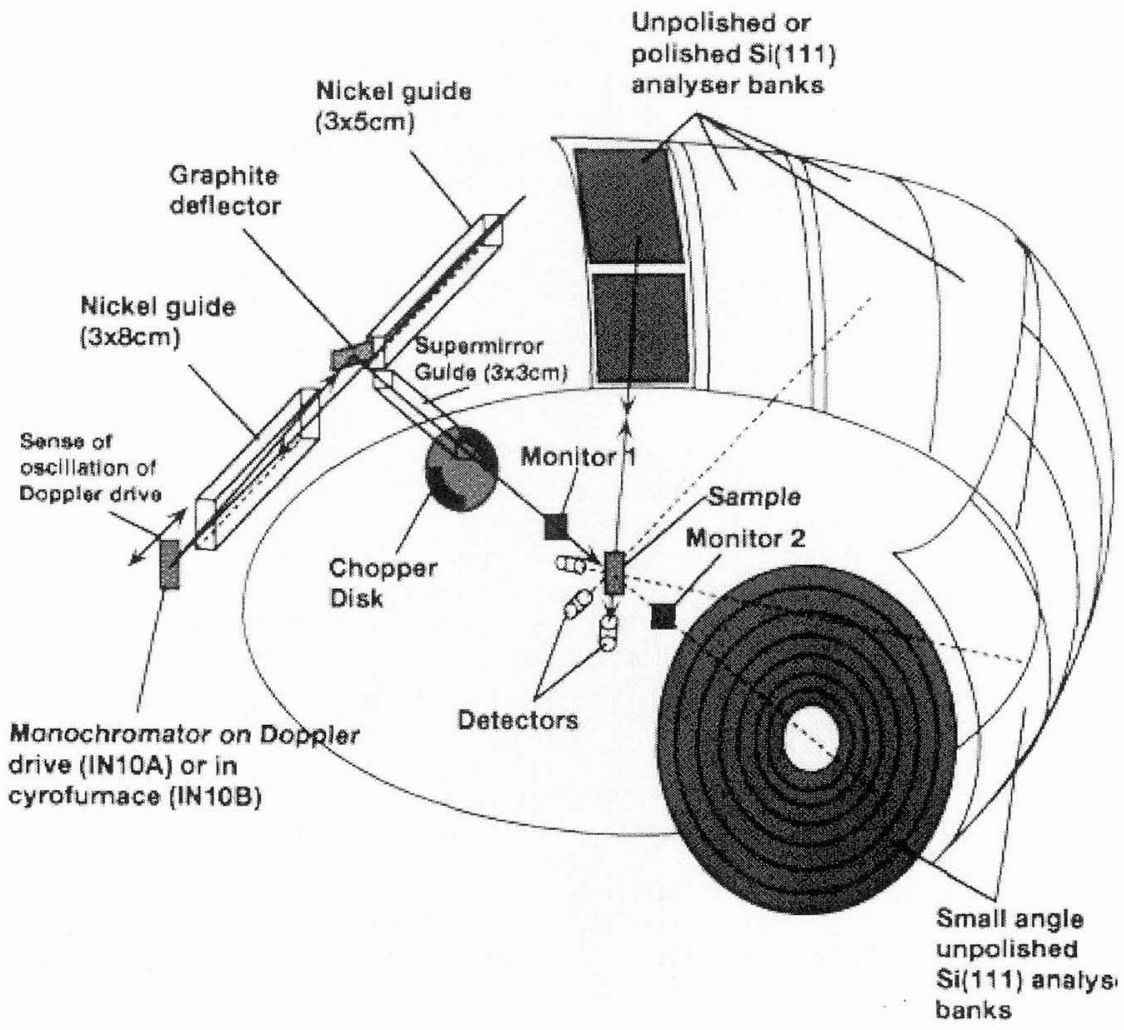

Figure 4 : Le spectromètre IN10 de l'ILL.

La gamme de transferts de moment $(Q)$ accessibles avec les cristaux de $\operatorname{Si}(1,1,1)$ varie entre 0 et $2 \AA^{-1}$. La limite supérieure est donnée par la relation $Q \max =(4 \pi / \lambda) \sin \left(\theta_{\max }\right), 2 \theta_{\max }\left(\sim 160^{\circ}\right)$ étant l'angle maximal de détection. Un monochromateur et des analyseurs composés de cristaux de silicium coupés dans la plan $(3,1,1)$ sont également disponibles. De part la longueur d'onde qu'ils selectionnent $(\lambda \approx 3.25 \AA)$, ils permettent une exploration beaucoup plus lointaine de l'espace réciproque (jusqu'à $3.8 \AA^{-1}$ ). Le flux est comparable à celui du Silicium $(1,1,1)$ poli, la fonction de résolution a une largeur de $3.5 \mu \mathrm{eV}$ et le transfert d'énergie maximum atteind $30 \mu \mathrm{eV}$. L'inconvénient majeur de cette configuration est la contamination des spectres par des réflexions de Bragg de l'aluminium qui apparaissent lorsque la longueur d'onde est inférieure à $5 \AA$. Les pics de Bragg provenant de l'échantillon posent également un problème lors de l'analyse de l'intensité diffusée. Un système de caches de cadmium peut être installé sur les analyseurs afin de masquer ces contributions.

Quand les cristaux du monochromateur et des analyseurs sont de même nature, le pic élastique se retrouve au centre de la fenêtre en énergie. Cette position correspond à une vitesse nulle du monochromateur. La position du pic élastique peut être déplacée lorsqu'on introduit une différence entre les distances interréticulaires du monochromateur et des analyseurs. Ce déplacement peut être introduit de façon controllée en dopant le silicium avec du germanium. Il existe ainsi un monochromateur du type, Si 0.9 Ge 0.1, pour lequel le pic élastique se retrouve en bord de fenêtre, la gamme accessible passant alors de $\pm 17 \mu \mathrm{eV}$ à $-32 \rightarrow 2 \mu \mathrm{eV}$. Le flux est légèrement inférieur à celui de la configuration normale $(60 \%)$. Un résumé des énergies, accessibles selon la nature du couple monochromateur / analyseur est présenté sur la figure 1.

Une autre façon de modifier l'énergie incidente consiste à faire varier le paramètre de maille du monochromateur en changeant sa température (de $80 \mathrm{~K}$ à $1000 \mathrm{~K}$, configuration IN10b). Tous les cristaux ne peuvent pas être utilisés comme monochromateur. Ceux-ci doivent posséder une dilatation thermique suffisament grande pour provoquer des variations en ênergie mesurables. Les cristaux ioniques $(\mathrm{KCl}, \mathrm{NaCl}$, $\mathrm{AgBr} . .$.$) offrent des caractéristiques intéressantes, et permettent d'augmenter la largeur de la fenêtre jusqu'à$ $140 \mu \mathrm{eV}$. Si la distance $d_{\text {monochromateur }}$ devient égale à $d_{\text {analyseurs }}$ pour une température comprise entre 80 et 
$1000 \mathrm{~K}$, le spectre final comprendra le pic élastique. Ceci est le cas pour le monochromateur de $\mathrm{KCl}(2,0,0)$ qui permet de mesurer des transferts d'énergie de -20 à $120 \mu \mathrm{eV}$ (tout en conservant une très bonne résolution de l'ordre de $1.5 \mu \mathrm{eV}$ ). Cette configuration est particulièrement intéressante, puisqu'elle réduit l'écart spectral entre les techniques de $\mathrm{RD}$ et de $\mathrm{TdV}$. Le cristal de $\mathrm{KCl}$ a un flux comparable à la configuration normale. D'autres exemples sont présentés dans le tableau I. Contrairement aux mesures réalisés à l'aide de la machine Doppler, les spectres mesurés avec le monochromateur sont obtenus point par point (transfert d'énergie par transfert d'énergie ?)

\subsection{IN13 [16]}

La dépendance en $\mathrm{Q}$ de l'intensité diffusée donne des informations sur la géométrie des mouvements moléculaires. C'est un des aspects particuliers à la diffusion neutronique. La longueur d'onde typique des spectromètres de type $\mathrm{RD}$ (configuration $\mathrm{Si}(111)$ ) limite $Q_{\max }$ à $2 \mathrm{~A}^{-1}$, ce qui peut s'avèrer insuffisant lorsqu'il s'agit de trancher entre différents modèles dynamiques. IN13 est un spectromètre installé sur un guide de neutrons thermiques, et fonctionne avec un monochromateur à température variable (cf in 10b). Afin d'augmenter le flux, on impose un gradient thermique perpendiculairement à la surface du cristal. Le monochromateur et les analyseurs sont constitués de cristaux de $\mathrm{CaF}_{2}(4,2,2)$, la longueur d'onde est de 2.23 $\AA$ et $Q_{\max }=5 \AA^{-1}$. L'inconvénient d'une telle longueur d'onde est la présence de pics de Bragg de l'aluminium et de l'échantillon. Cette diffusion cohérente est gênante pour l'analyse de la diffusion incohérente (voir section $2.2 \ldots$ ). Plus que les autres spectromètres à RD, IN13 est adapté à l'étude des échantillons non-cristallins (polymères, biomolécules ...).

Tableau I. : Comparaison du flux au niveau de l'echantillon (les valeurs sont exprimées en fonction du flux mesuré sur IN10 dans la configuration classique (flux=10) avec des cristaux de $\mathrm{Si}(111)$ non-polis), de la résolution et de la gamme spectrale accessible pour les différents instruments (de l'ILL). Un tableau plus complet concernant les monochromateurs de IN10b se trouve dans [3], page 60 .

\begin{tabular}{|c|c|c|c|}
\hline $\begin{array}{c}\text { Instrument } \\
\text { (monochromateur) }\end{array}$ & Flux & $\begin{array}{c}\text { Résolution } \\
\text { (FWHM) }\end{array}$ & $\begin{array}{c}\text { Gamme spectrale } / \\
\mu \mathrm{eV}\end{array}$ \\
\hline $\mathrm{IN} 10$ (Si(111) non-polis) & 10 & 0.8 & \pm 16 \\
\hline $\mathrm{Si}(111)$ polis & $2-3$ & 0.3 & \pm 16 \\
\hline $\mathrm{Si}(311)$ & $2-3$ & 3.5 & \pm 30 \\
\hline $\mathrm{Si}(0.9) \mathrm{Ge}(0.1)$ & 7 & 0.8 & $-30,+2$ \\
\hline $\mathrm{KCl}(2,0,0)$ & 12 & 1.5 & $-20,+120$ \\
\hline $\mathrm{NaCl}(2,0,0)$ & 15 & 1.5 & $-520,-320$ \\
\hline $\mathrm{NaF}(1,1,1)$ & 3 & 1.5 & $-810,-600$ \\
\hline $\mathrm{N} 16(\mathrm{Si}(111)$ non-polis) & 40 & 0.8 & \pm 15 \\
\hline $\mathrm{Si}(111)$ polis & 10 & 0.3 & \pm 15 \\
\hline $\mathrm{IN} 13(\mathrm{CaF}(4,2,2))$ & $2-3$ & 8 & $-125,+300$ \\
\hline
\end{tabular}

\subsection{IN16 [17,18]}

Ce spectromètre est le plus récent à l'ILL. Le point faible de tous spectromètres de type RD est le faible flux de neutrons. IN16 a été construit en vue d'améliorer ce problème. La dimension du faisceau incident extrait du guide primaire est relativement importante $(6 \mathrm{~cm} \times 12 \mathrm{~cm})$. Grâce à une optique focalisante (guide, monochomateur), il est alors possible de concentrer ces neutrons au niveau de l'échantillon $(3 \mathrm{~cm} x 3 \mathrm{~cm})$ et d'augmenter ainsi considérablement le flux incident. Le monochromateur est de taille assez importante (20 $\mathrm{cm} \times 45 \mathrm{~cm}$ ) comparé aux dimensions d'IN10 $(3 \mathrm{~cm} \times 8 \mathrm{~cm})$ et se présente sous la forme d'une calotte sphérique de $2 \mathrm{~m}$ de rayon. Malgré les problèmes de poids $(5.5 \mathrm{~kg})$, une machine Doppler bien équilibrée 
permet de le déplacer à $14 \mathrm{~Hz}$ sur une distance de $5 \mathrm{~cm}$. Dans ces conditions, un transfert maximal d'énergie de $\pm 14 \mu \mathrm{eV}$ peut être atteind, avec une résolution de $0.9 \mu \mathrm{eV}$. Le flux de neutrons sur l'échantillon est $\sim 3$ fois supérieur à celui d'IN10 tandis que l'efficacité du spectromètre secondaire permet d'obtenir un comptage $\sim 4$ fois supérieur. Ces valeurs concernent la configuration normale: monochromateur et analyseurs de $\mathrm{Si}(1,1,1)$ non-polis et déformés. Il existent également deux autres configurations à plus haute résolution qui permettent d'atteindre $0.4 \mu \mathrm{eV}$ et $0.25 \mu \mathrm{eV}$ (FWHM), toujours au détriment du flux d'un facteur 2 à 4 .

\subsection{IRIS [19]}

Le réacteur de l'ILL est une source continue de neutrons. Chaque instrument de type RD hache le faisceau incident afin de permettre une analyse TdV des neutrons diffusés. A ISIS (Royaume-Uni) la source est de type spallation et des bouffés de neutrons sont produites à une fréquence de $50 \mathrm{~Hz}$. Sur l'instrument de RD, IRIS (fig. 5), la structure spectrale des bouffés remplace le rôle de la machine Doppler ou le monochromateur à température variable. La gamme de longueurs d'ondes de chacune des bouffés est sélectionnée à l'aide de deux choppers. Le transfert d'énergie est toujours déterminé par analyse TdV (correspondant au trajet modérateur-échantillon-analyseur-détecteur). L'avantage de cette technique est qu'une gamme importante de transferts d'énergie peut être mesurée simultanément. Elle peut atteindre 5 $\mathrm{meV}$ avec une résolution (déterminée par les analyseurs de graphite) de $50 \mu \mathrm{eV}$. Avec les analyseurs de mica, la résolution atteint $1 \mu \mathrm{eV}$ tandis que le transfert d'énergie maximum est de $300 \mu \mathrm{eV}$. Dans cette deuxième configuration, le comptage de neutrons est relativement faible en raison de la pauvre reflectivité des analyseurs et au nombre limité de neutrons de très grande longueur d'onde $(\sim 18 \AA)$ dans le faisceau incident. La configuration habituelle utilise les analyseurs de graphite. En terme de la résolution, ce type de spectromètre se situe entre le TdV classique (IN5) et la RD décrite ci-dessus.

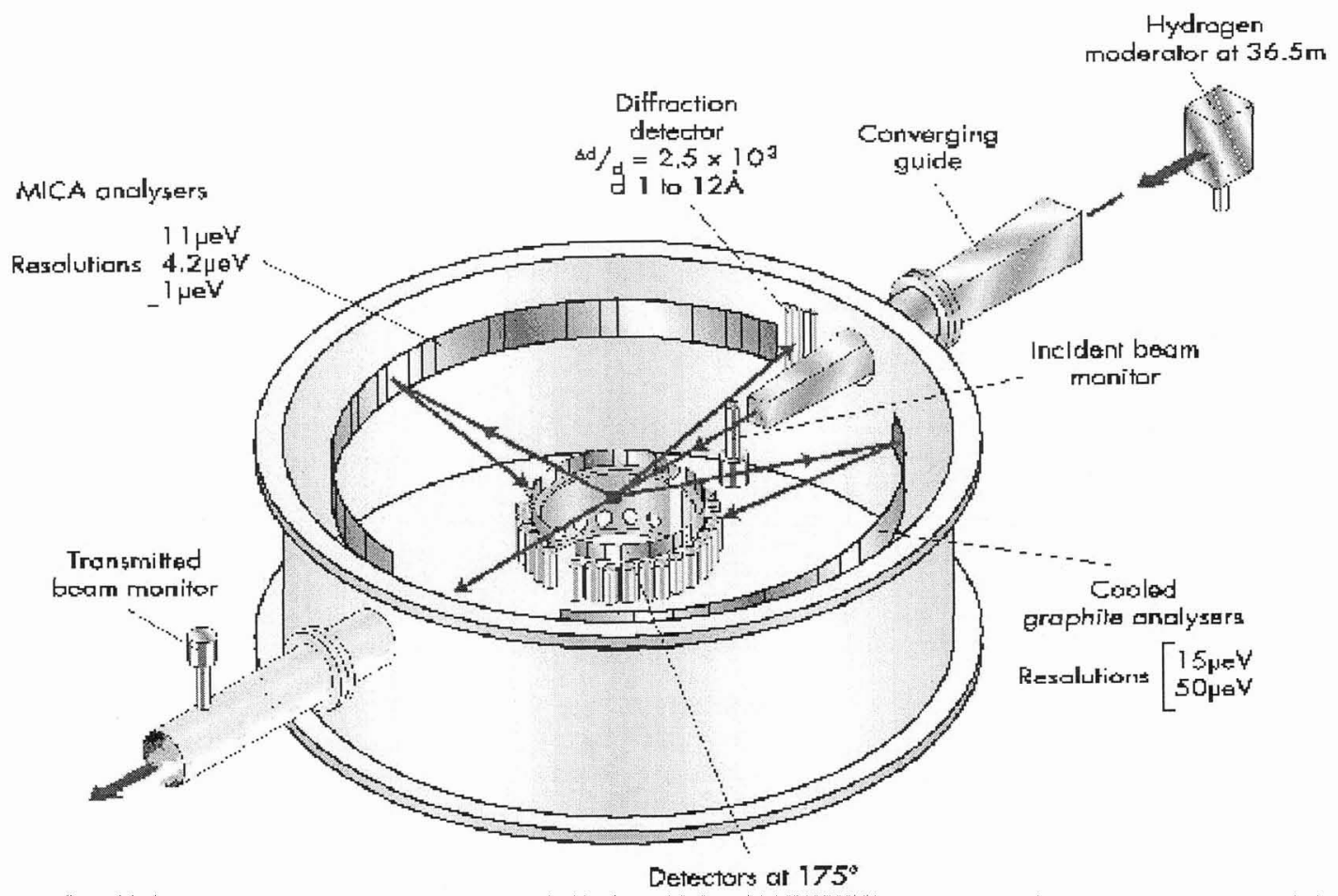

Figure 5 : Le spectromètre IRIS de ISIS 
Bien que les neutrons soient produits par bouffés, les choppers selectionnent $\delta \lambda$ de telle sorte qu'ils arrivent de façon continue sur l'échantillon. Les détecteurs doivent être cachés des neutrons qui ne parcourent pas le chemin adéquate (échantillon-analyseur-détecteur). Ceci est réalisé en les positionnant en contrebas de l'échantillon, du côté "analyseurs". Cette configuration détecteurs/analyseurs permet d'utiliser les neutrons diffusés sur 360 degrés, et augmente ainsi le comptage des neutrons (facteur 2).

\section{EXEMPLES D'EXPERIENCES}

La plupart des expériences réalisées sur les spectromètres cités auparavant sont de type quasiélastique. La haute résolution permet l'étude de la dynamique diffusive sur une échelle de temps de la nanoseconde. Du fait de la faible largeur de la fenêtre en énergie, il est nécessaire de connaitre à prioris le domaine de températures (de pression ou de tout autre facteur externe) dans lequel on s'attend à trouver un signal. Nous verrons que les scans élastiques permettent de sonder rapidement ces conditions. L'effet tunnel des groupements de protons, les excitations magnétiques, ainsi que et les excitations de type roton de l'helium 4 font également l'objet d'un grand nombre d'expériences inélastiques sur ce type de spectromètre.

\subsection{Le scan élastique}

Dans ce type de scan, l'énergie des neutrons incidents est fixe, et correspond à celle déterminée par les analyseurs. Il suffit pour cela d'arrêter la machine Doppler (à condition bien entendu que le même type de cristal soit utilisé pour le monochromateur et les analyseurs). Ceci peut Également être réalisé avec un monochromateur à température variable pour une température $T$ telle que $d_{\text {mono }}(T)=d_{\text {analyseurs. }}$. Dans ces conditions, le transfert d'énergie est nul, et l'on peut faire varier certains paramètres expérimentaux tels que la température, le pression.... L'avantage de ce type de scan est qu'il permet de sonder rapidement l'évolution de la dynamique du système sur une plage importante de température. En général l'intensité élastique décrôt lorsque la température augmente, au cause des effets de type Debye-Waller, $I(T)=I(0) \exp (-$ $Q^{2} u(T)^{2}$ ) (où $u$ est le déplacement carré moyen des atomes). Une dynamique moléculaire sur l'échelle de temps de la nanoseconde, thermiquement activée, provoque également une baisse d'intensité élastique à cause du facteur de structure dynamique. Un changement de pente de l'évolution de l'intensité élastique est observé à la température où le signal quasiélastique devient plus important que la résolution $\left(\tau_{\mathrm{c}}^{-1} \approx 10^{9} \mathrm{~s}^{-1}\right)$. De même, des modifications sont attendues lorsque le signal quasiélastique devient trop large $\left(\tau_{c}^{-1}>10^{10} \mathrm{~s}^{-1}\right)$ et que sa contribution au pic élastique se trouve négligeable. Bien qu'un scan élastique permet en théorie de déterminer $u(T)$, l'énergie d'activation d'un processus ainsi que le facteur de structure élastique incohérente (EISF), il est surtout utilisé pour identifier la gamme de températures où la diffusion quasiélastique peut être mesurée. Deux exemples sont énoncés ci-dessous.

En général les polymères montrent un comportement dynamique très complexe. Les mouvements s'étendent sur une échelle temporelle et spatiale très vaste. La technique de rétrodiffusion est largement utilisée pour étudier les mouvements locaux ainsi que la transition vitreuse dans ces systèmes. La figure 6 montre un scan élastique de poly isoprène (monomère $\left[-\mathrm{CH}_{2}-\mathrm{C}\left(\mathrm{CH}_{3}\right)=\mathrm{CH}-\mathrm{CH}_{2}\right]$ ) [20]. Les mouvements locaux, tels que la rotation des groupements méthyles et les mouvements de la chaîne apparaissent clairement. A basse température, l'intensité élastique décroît d'abord faiblement puis diminue plus rapidement à partir de $100 \mathrm{~K}$. Par deutération sélective du rotateur méthyle et de la chaîne, il est possible d'observer des contrastes importants dans l'intensité diffusée. Il devient alors évident que cette perte d'intensité est liée aux rotations des groupements méthyle. Après une première saturation, l'intensité décroît à nouveau lors de la transition vitreuse $\left(220 \mathrm{~K}<T \approx T_{g}<290 \mathrm{~K}\right.$ ) où la chaîne devient mobile. Comme pour beaucoup d'autres substances vitreuses, le degel rencontré vers $T_{g}$ est décrit par deux processus: une relaxation $\alpha$, liée à la dynamique segmentale rapide des polymères, et une relaxation $\beta$ plus lente. C'est typiquement entre 30 et $50 \mathrm{~K}$ au dessus de $T_{g}$ que l'élargissement quasiélastique des spectres liée à la 'relaxation $\alpha$ ', est observable [21]. 


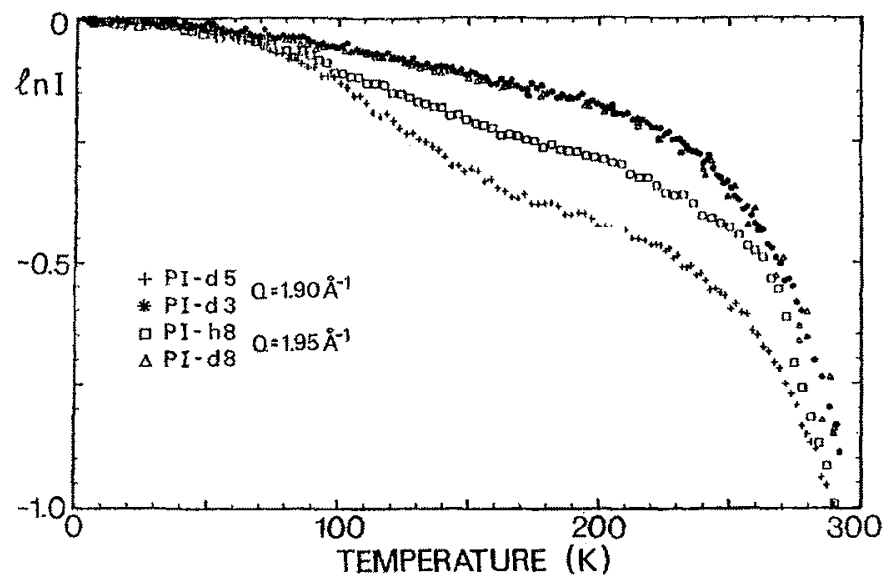

Figure 6 : Scans élastiques de poly-isoprène. L'effet de contraste entre les échantillons partiellement deutérés permet d'identifier la contribution des groupements méthyles au delà de $100 \mathrm{~K}$.

Le radical nitroxyde $\mathrm{C}_{9} \mathrm{H}_{16} \mathrm{NO}_{2}$ (tano) forme des composés d'inclusion avec une grande variété de chaînes linéaires (octane...). Les molécules de tano s'organisent de façon à créer des canaux dans lesquels les molécules d'octane viennent se loger. A température ambiante, ce système est dynamiquement désordonné. Outre le mouvement des molécules incluses (autour et le long des canaux), les molécules de tano peuvent adopter l'une ou l'autre de ses deux formes énantiomères par inversion dynamique du cycle pipéridinique. Lors d'une descente en température, une modification de l'intensité élastique apparait vers $170 \mathrm{~K}$ (figure 7 (a)). Cette modification est associée à l'apparition d'une transition ordre / désordre modifiant la dynamique des molécules de tano. Cette transition du premier ordre admet un large hystérésis et apparait à $250 \mathrm{~K}$ lors de la remonté en température. La dynamique du système n'est donc pas la mêrne selon les traitements thermiques que l'on fait subir à l'échantillon. Les modifications de l'intensité élastique observées sur le cycle complet en température sont en parfait accord avec les températures de transition déterminées par DSC et diffraction. A partir de l'évolution de l'intensité élastique avec le vecteur de diffusion il est possible de calculer le déplacement carré moyen pour chaque température (figure $6(b)$ ). Ce calcul n'est valable que pour plus basses températures. Aux plus hautes températures, l'apparition d'une contribution quasilélastique est responsable d'une diminution de l'intensité élastique. Cela se traduit en apparence par une augmentation plus ou moins importante du déplacement carré moyen. Une fois de plus, les transitions de phase ainsi que l'hystérésis sont clairement visibles. Ces observations effectuées par $\mathrm{RD}$ confirment l'origine dynamique de la transition de phase.

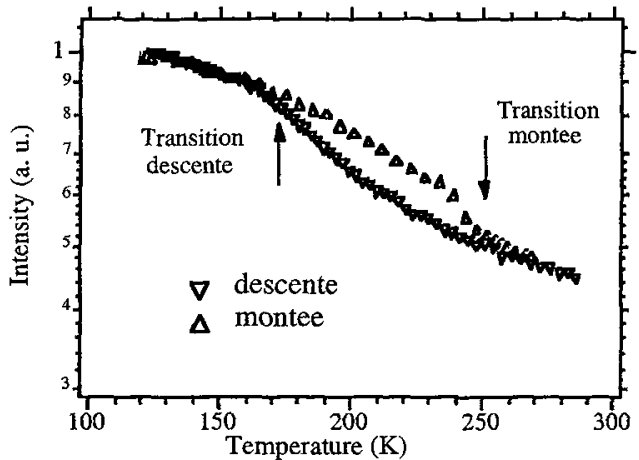

(a)

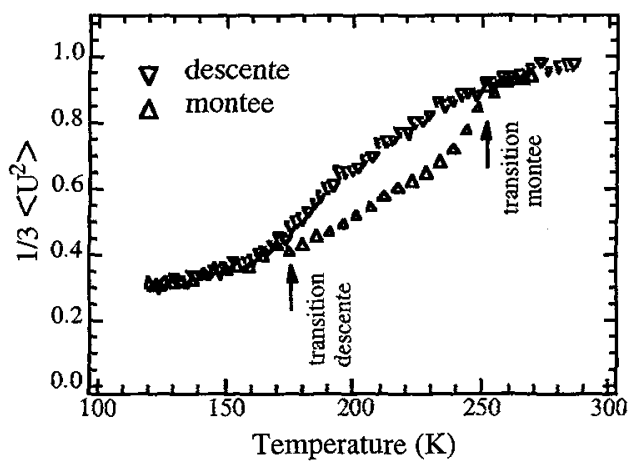

(b)

Figure 7 : (a) Scan élastique du système tano-octane réalisé sur le spectromètre à rétrodiffusion INI6. (b) Déplacement carré moyen correspondant. 


\subsection{Le scan quasiélastique}

Ce type d'étude permet de déterminer les temps caractéristiques des mouvements ainsi que leur évolution avec la température. Dans certains cas, il est également possible de déduire leur géométrie.

La dynamique des polymères sous pression. Selon la théorie du couplage des modes, le paramètre dominant la relaxation autour de $T_{\text {e }}$ est la densité, quantité qui peut varier avec la température et la pression. Il devient alors très intéressant d'étudier la transition vitreuse en fonction de la pression hydrostatique externe [22]. L'effet de la pression sur la dynamique des chânes à une température au dessus de $T_{8}$ est dramatique. Un exemple est donné dans figure 8 dans le cas du polybutadiène (monomère: $\left[-\mathrm{CH}_{2}-\mathrm{CH}=\mathrm{CH}-\mathrm{CH}_{2}-\right]$ ) [23] . A $300 \mathrm{~K}$ et à pression ambiante, la dynamique est presque trop rapide pour la fenêtre en energie. Le signal est peu intense, et présente une forme spectrale non-Lorentzienne (phénomène caractéristique dans les verres). A plus haute pression le signal devient plus intense et la largeur de la contribution quasiélastique diminue sensiblement. A $3 \mathrm{kbar}$, on se rapproche encore de la résolution. Lors de l'étude de la dynamique des polymères, il est indispensable de compléter les mesures de RD (scan élastique et quasiélastique) par des mesures TdV et EdS afin d'évaluer le rôle de la température et de la pression hydrostatique sur la dynamique globale du composé [24].

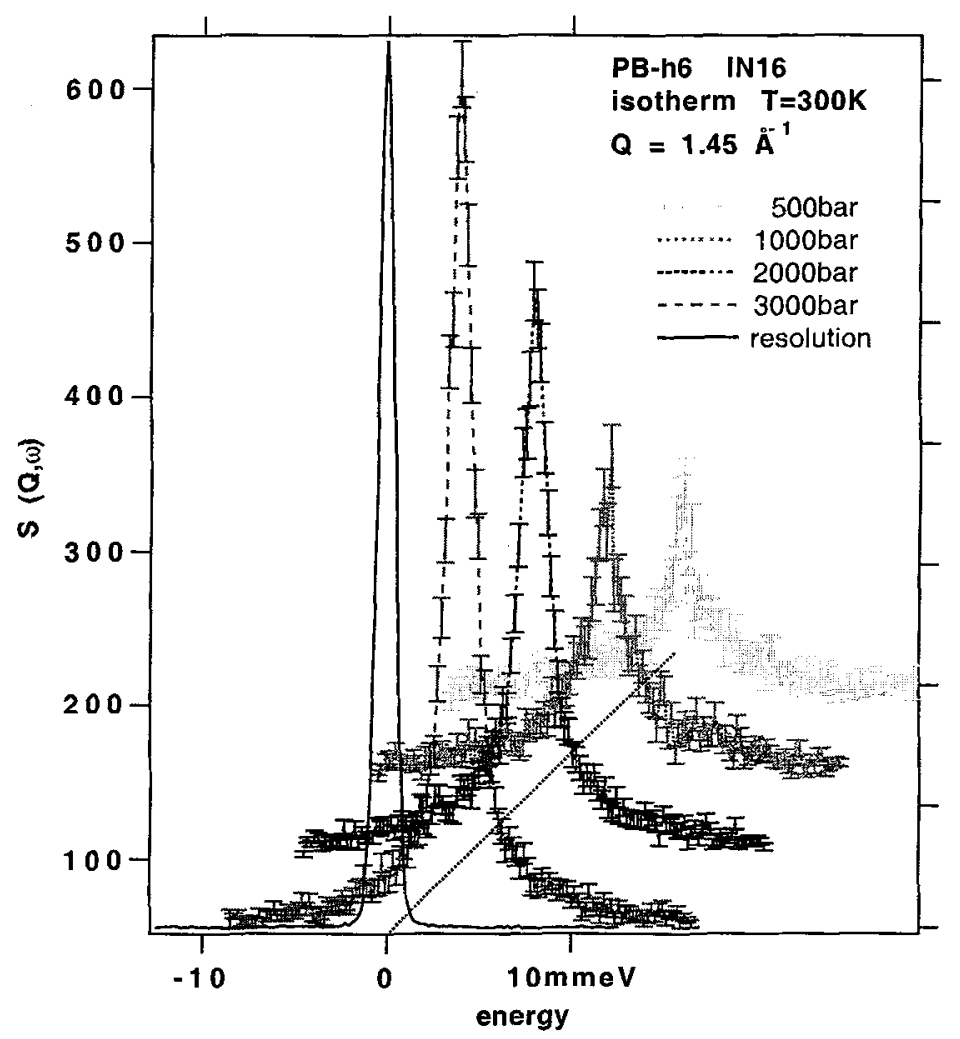

Figure 8 : Spectres quasiélastiques de polybutadiène mesurés en fonction de la pression hydrostatique.

Le transfert de proton le long d'une liaison hydrogène est l'exemple le plus simple d'une réaction chimique. Dans le cas de l'acide benzoïque $\left(\mathrm{C}_{6} \mathrm{D}_{5} \mathrm{COOH}, \mathrm{BA}\right)$, deux molécules sont liées par une paire de liaisons hydrogène $(-\mathrm{O}-\mathrm{H} \ldots \mathrm{O}=)$, engendrant des dimères en phase condensée. En l'absence d'interactions inter-dimères, il existe deux configurations (tautomères) stables du dimère : $\mathrm{H}_{\mathrm{L}}^{1} \mathrm{H}_{\mathrm{R}}^{2}$, où le proton 1 est lié à la molécule $\mathrm{L}$, et $\mathrm{H}^{2}{ }_{\mathrm{L}} \mathrm{H}^{1}$. Les deux protons sont transférés de façon concertée le long des liaisons hydrogène entre les molécules. A l'état solide, les tautomères sont énergétiquement inéquivalents: il existe un 
tautomère stable et un tautomère métastable peuplé à plus haute température. Une étude très complète de la dynamique du transfert de proton, réalisée par RMN, a permis de déterniner la gamme de températures adaptée à des mesures par RD [8]. La figure 9(a) montre l'évolution de la diffusion quasiélastique entre 50 $\mathrm{K}$ et $110 \mathrm{~K}$. Les temps de corrélation $\tau_{c}^{-T}(T)$ mesurés par les deux techniques sont présentés sur la fígure 2. La RMN mesure le mouvement atomique au travers des interactions dipolaires entre les spins nucléaires. Les mesures neutroniques permettent d'étalonner ces interactions.

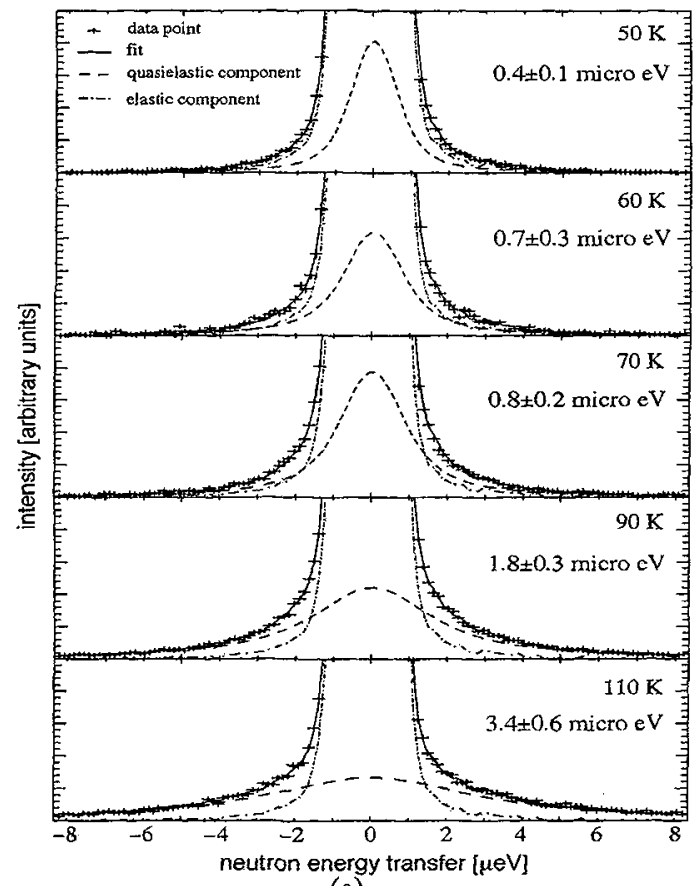

(a)

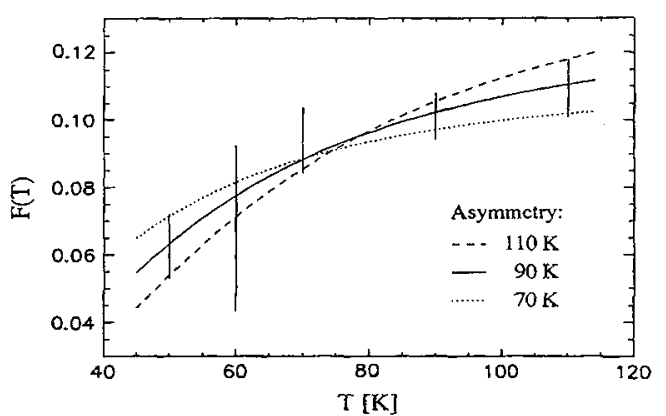

(b)

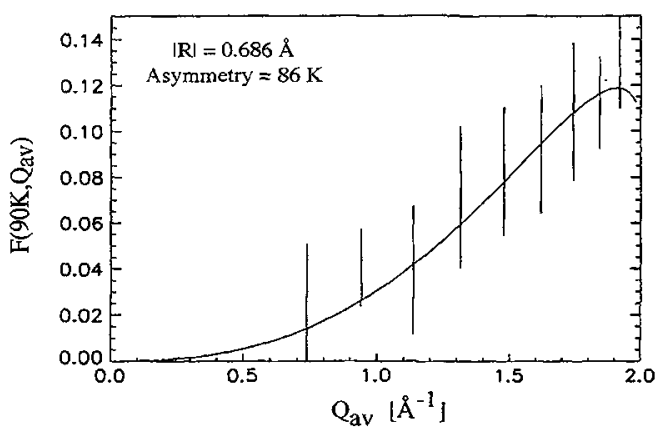

(c)

Figure 9 : (a) Spectres quasiélastiques de BA, mesurés sur IN16 dans la configuration habituelle. Les largeurs (HWHM) sont indiquées pour chaque température. (b) Dépendance en température de l'intensité quasiélastique. (c) Dépendance en $Q$ de l'intensité quasiélastique.

Un échantillon composé de 9 monocristaux alignés a été utilisé afin d'éteindre tous les pics de Bragg et de simplifier l'analyse de l'intensité incohérente (liée à la liaison hydrogène). A une valeur constante de $Q$, la dépendance en température de l'intensité quasiélastique est liée au peuplement du tautomère métastable. II est alors possible de déduire la différence d'énergie entre les deux tautomères à partir de cette variation d'intensité (voir fig. 9(b)). A température constante, la dépendance en $Q$ de l'intensité quasiélastique permet de déterminer la distance parcourue par les protons entre les sites correspondant aux deux formes 
tautomèriques (voir fig. 9(c)). Une valeur de $0.7 \AA$ a ainsi été déterminée, et confirme que le mouvement observé se produit le long des liaisons hydrogènes plutot que par par rotation des groupements acides.

\subsection{Le scan inélastique}

Il est également possible de mesurer des excitations inélastiques jusqu'à $1 \mathrm{meV}$ avec une résolution de l'ordre de $1 \mu \mathrm{eV}$. Quelques exemples sont cités ci-dessous.

L'effet tunnel de rotateurs moléculaires (groupements méthyles, méthane, ammonium) conduit à des éclatements inférieurs à $1 \mathrm{meV}$ des niveaux vibrationnels [25]. La technique de RD a permis de compiler un grand nombre de mesures d'éclatement tunnel [26]. L'ensemble de ces données est désormais exploité pour caractériser avec une précision inégalée, les interactions intermoléculaires à l'origine de la barrière d'énergie potentielle de rotation (l'éclatement tunnel dépendant de façon exponentielle de l'amplitude et de la largeur de cette barrière). Trois exemples sont ennoncés ci-dessous.

L'acétanilide est une molécule où un groupement méthyle est lié directement à un groupement peptidique, ce dernier étant responsable de la présence de liaisons hydrogènes intermoléculaires. L'éclatement tunnel du rotateur méthyle permet alors de sonder la distribution électronique le long des liaisons hydrogènes [27]. La figure 10 montre le spectre mesuré à $2 \mathrm{~K}$ sur IN16. Une simple soustraction de la fonction de résolution révèle deux pics en $\pm 0.3 \mu \mathrm{eV}$. Le spectre a été mesuré dans une configuration de plus haute résolution. La fonction d'appareil asymétrique est liée à une grande différence entre la résolution du monochromateur et celle des analyseurs.

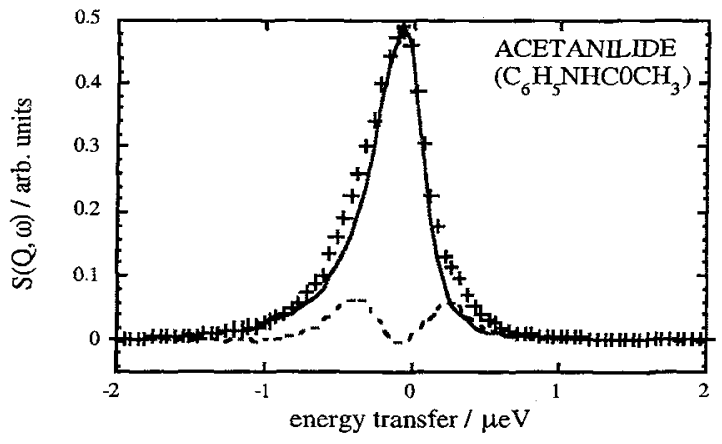

Figure 10 : Le spectre de l'effet tunnel du groupement méthyle de acétanilide, mesuré sur IN16 avec la meilleurerésolution possible

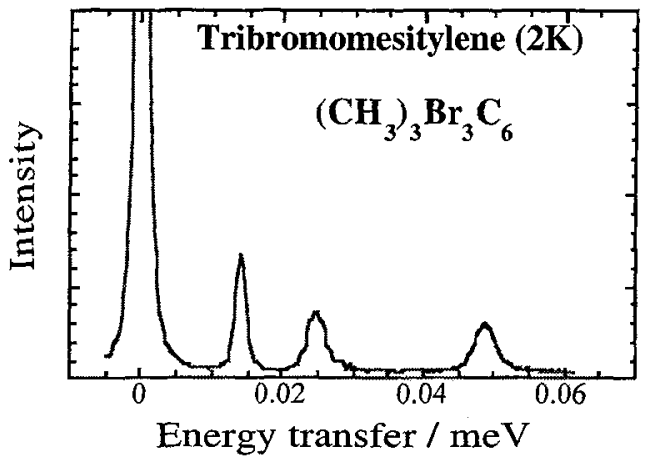

Figure 11: Spectre de l'effet tunnel des trois groupements méthyles de tribromomésitylène, mesuré sur IN10b avec le monochromateur $\mathrm{KCl}(2,0,0)$. 
La molécule isolée de tribromomésitylène possède trois groupements méthyles équivalents. Ce n'est plus le cas en phase condensée en raison de des interaction intermoléculaires. Les expériences réalisées sur IN10b avec le monochromateur de $\mathrm{KCl}(2,0,0)$ (fig. 11) montre l'apparition de trois pics tunnels relativement bien séparés (à $15 \mu \mathrm{eV}, 25 \mu \mathrm{eV}$ et $49 \mu \mathrm{eV}$ ). Ces observations fournissent des informations précises sur la nature des interactions intermoléculaires.

Le dernier exemple concerne l'effet tunnel dans la $\gamma$-picoline. Les études structurales ayant pu mettre en évidence un seul type de groupement methyle, on s'attendait à ne trouver qu'un seul pic tunnel. Les résultats ont été beaucoup plus surprenants. Par des techniques de TdV, trois pics ont été observés autour de $500 \mu \mathrm{eV}$ [28]. La même expérience réalisée sur IN10b avec une bien meilleure résolution (monochromateur $\mathrm{NaCl}$ $(2,0,0)$ ), a révélé une structure spectrale pour chacun d'entre-eux (fig. 12) [29]. Cette structure complexe est l'évidence d'une dynamique quantique où les rotateurs méthyles sont couplés entre-eux : la rotation de chaque méthyle entrainant un mouvement translationnel du centre de masse des rotateurs.

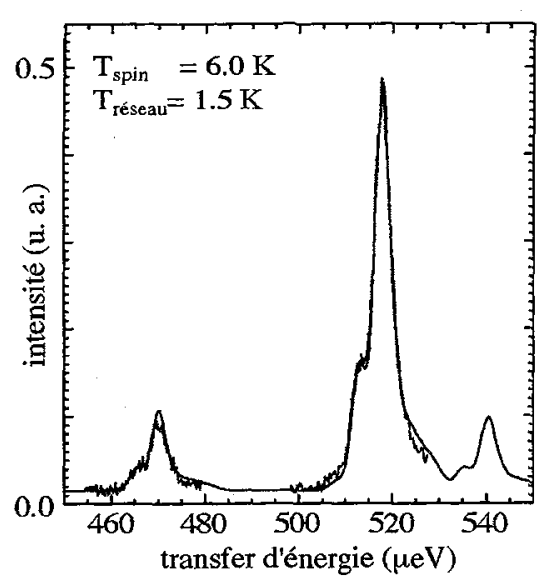

Figure 12 : Spectre de l'effet tunnel de la $\gamma$-picoline, mesuré sur IN10b avec le monochromateur de $\mathrm{NaCl}(2,0,0)$. La courbe contimue est le résultat d'une modélisation numérique de dynamique quantique.

Le monochromateur $\mathrm{NaF}(1,1,1)$ permet d'aller encore plus loin dans les transferts d'energie $(\Delta \mathrm{E} \sim 800$ $\mu \mathrm{eV})$ tout en conservant une résolution de l'ordre de $1 \mu \mathrm{eV}$. Il a été utilisé avec succés pour mesurer l'énergie des excitations de type "roton" dans l'hélium 4 ainsi que leur durée de vie en fonction de la température (fig. 13(a) [30]. Si la durée de vie mesurée est en accord avec des mesures d'écho de spins et la théorie de Bedell (fig. 13(b)), il n'en est pas de même pour l'évolution de l'énergie avec la température (plus faible que les valeurs prédites) (fig. 13(c)). Ces mesures de grandes précisions semblent remettre en cause la théorie jusqu'alors établie. 


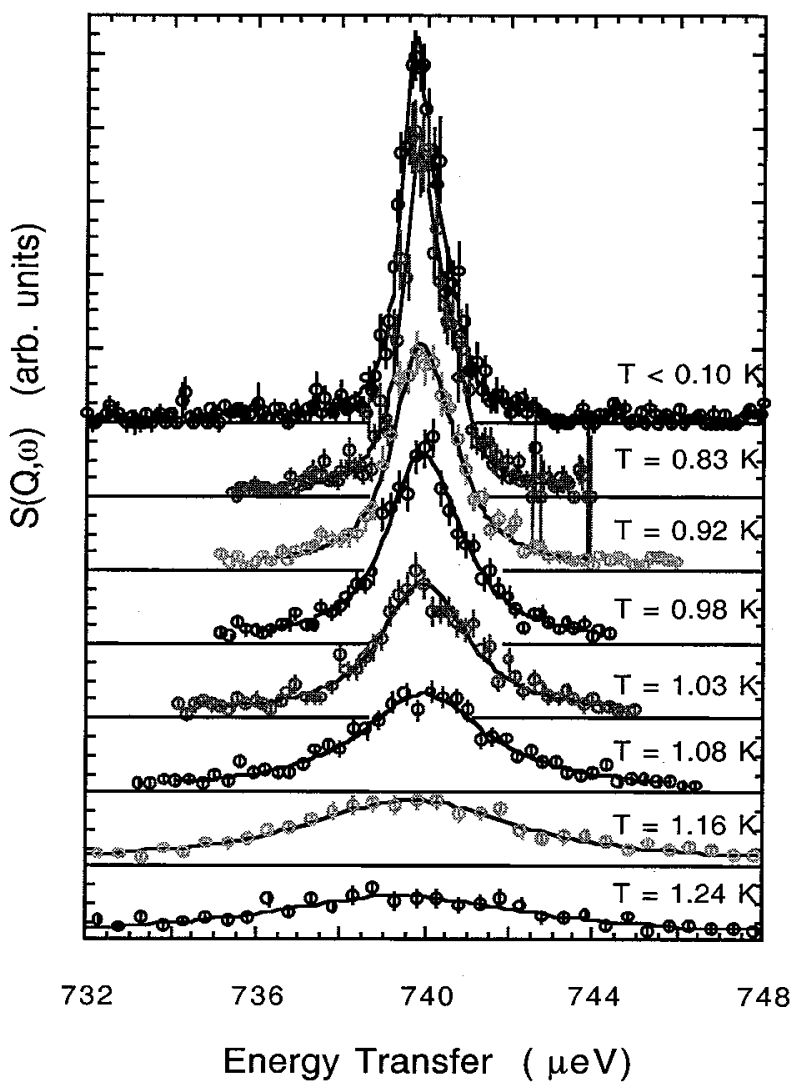

(a)

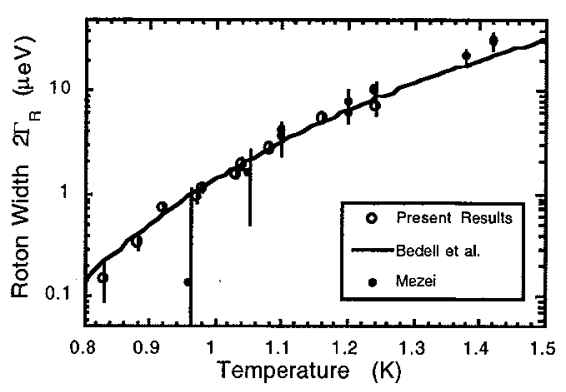

(b)

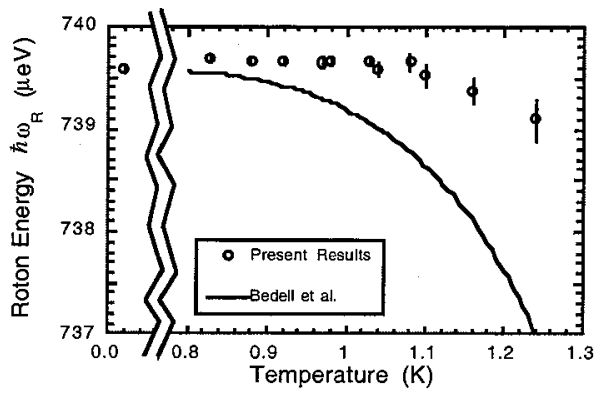

(c)

Figure 13: (a) Excitations de type roton mesurées sur IN10b avec le monochromateur $\mathrm{NaF}(1,1,1)$. (b) Largeur de l'excitation en fonction de la température. (c) Energie de l'excitation en fonction de la température. 


\section{L'ANALYSE DES DONNEES : LES PROBLEMES ASSOCIES}

La première étape de l'analyse consiste à réduire les données, c'est à dire, à corriger le signal enregistré de toutes les contributions expérimentales (bruit de fond, absorption et self screening liés à la géométrie de l'échantillon, efficacité des détecteurs, angle solide de détection...). Le but de la réduction est de déterminer la fonction de diffusion réelle $S(Q, \omega)$, quantité directement comparable à des prédictions théoriques. Le programme couramment utilisé à l'ILL pour corriger les données de rétrodiffusion s'appelle SQ $\omega$. Afin de réaliser chacune de ces corrections, il est nécessaire d'effectuer des mesures complémentaires. Pour corriger les données du bruit de fond, on enregistre le signal provenant d'une cellule vide. Dans certains cas, le système est formé d'un substrat contenant d'autres types de molécules (système graphite/méthane, zéolithe/alcane...). Afin d'accéder à la dynamique des molécules incluses, la cellule vide doit être remplacée par le substrat lui même. Afin d'accéder à la fonction de diffusion $S(Q, w)$ globale, il est nécessaire de normaliser l'intensité de chaque détecteur. On enregistre pour cela l'intensité diffusée par un échantillon étalon: le vanadium. Dans la gamme de longueurs d'onde utilisée, celui-ci présente le grand avantage de diffuser de manière totalement incohérente. L'intégration du pic élastique (après soustraction de bruit de fond et corrections d'absorption) sur chacun des détecteurs fournit alors des termes correctifs qui, appliqués à l'échantillon corrigent les problèmes d'efficacité, et d'angle solide de détection.

Les données réduites, ou corrigées, peuvent ensuite être dépouillées afin de caractériser la dynamique du système. Dans la pupart des expériences, le signal se présente sous la forme d'un pic élastique (limité par la résolution), et d'une contribution quasiélastique formée de lorentziennes centrées à l'origine. La largeur de la composante quasiélastique nous renseigne sur les temps caractéristiques des processus dynamiques tandis que l'évolution de la partie élastique (avec le vecteur de diffusion) donne des informations sur la géométrie des mouvements. L'analyse des données consiste en grande partie à séparer ces deux contributions. Le profil exacte de la fonction de résolution dépend du choix de la configuration. Même si il se rapproche d'une fonction gaussienne (configuration "basse" résolution), ou d'une lorentzienne (configuration "haute" résolution), il existe généralement de faibles déviations à ces fonctions théoriques. Aussi, la prise en compte de la résolution expérimentale dans le traitement des données se fait par convolution numérique. Celle ci peut être mesurée sur du vanadium ou sur l'échantillon lui même, à des températures suffisament basses pour que l'intensité diffusée soit totalement élastique.

Il existe à l'ILL, plusieurs programmes permettant de traiter les données. PROFIT est un programme qui permet d'affiner les données sur la base de contributions théoriques de forme delta, Gaussienne ou Lorentzienne, sans pour autant évoquer un modèle dynamique spécifique. Un autre programme, QUASILINE, basée sur la méthode d'entropie maximale, permet d'obtenir la probabilité pour les différentes composantes de forme Lorentzienne ainsi que les facteurs de structure incohérents (élastique et quasiélastique). Ceci constitute une approche élementaire nécessaire pour établir l'évolution des contributions principales avant de procéder à des dépouillements plus astucieux. AGATHE est un programme plus évolué qui permet l'analyse des spectres à partir d'un modèle microscopique (sauts sur $\mathrm{n}$ positions, diffusion à longue distance...). Il est également possible d'analyser simultanément des mesures réalisées sur différents spectromètre ou à différentes températures.

Plusieurs questions peuvent se poser lors du dépouillement des données.

Est ce que les données sont perturbées par des effets de diffusion multiple?

Durant l'analyse on suppose en général que les neutrons ne sont diffusés qu'une seule fois par l'échantillon. La quantité de matière est géneralement choisie pour atteindre une transmission de $90 \%$ (diffuseur $10 \%$ ). La probabilité de diffusion multiple (diffusion double) atteind dans ce cas $10 \%$ de l'intensité diffusée. Elle peut néanmoins prendre des proportions plus importantes dans les régions de l'espace où l'intensité diffusée (au premier ordre) est faible. Celle-ci peut être d'origine élastique-élastique, élastique-inélastique ou inélastique-inélastique. La diffusion multiple inélastique se manifeste par des pics inélastiques de faible intensité positionnés à des valeurs multiples du transfert d'énergie original. Pour la diffusion quasiélastique, elle apparait comme une modification de la forme du signal, ou encore comme une variation de la proportion elastique / quasiélastique. Ce type de problème est fréquement rencontré aux petits angles dans le cas des diffuseurs cohérents. Pour les expériences quasiélastiques, on observe très souvent que le facteur de structure élastique (EISF) ne tend pas vers 1 lorsque $Q$ tend vers 0 . De même, le facteur de structure quasiélastique (QISF) ne tend pas vers 0 pour des faibles valeurs de $\mathrm{Q}$. La diffusion multiple peut se manifester également par un désaccord entre l'intensité élastique, mesurée lors d'un scan élastique, et l'intensité obtenue lors des scans quasiélastiques, phénomène d'autant plus marqué à haute température 
lorsque les processus de diffusion élastique-inélastique deviennent plus important que les processus élastique-élastique.

La correction de la diffusion multiple n'est pas triviale et nécessite la connaisance à prioris de la loi de diffusion. Une stratégie "auto-consistente" est alors adoptée. Elle consite tout d'abord à dépouiller les spectres sur la base d'un modèle probable. On calcule ensuite la propabilité des processus de diffusion multiple et détermine les facteurs de correction à appliquer. L'étape suivante consiste à corriger le modèle et à réitérer l'opération. Pour chaque cycle, le modèle est modifié. Après 4 à 5 itérations, on converge vers la solution. Les programmes les plus connus pour ce type de correction sont DISCUS (installé à l'ILL) et MSCAT.

Est ce que l'intensité élastique est cohérente ou incohérente?

Bien que la longueur d'onde classique des spectromètres à RD soit relativement élevée $(6.27 \AA)$, des pics de Bragg peuvent apparaître pour un certain nombre d'échantillons cristallins (typiquement, lorsque les paramètres de maille dépassent $\lambda / 2$ ). A cause de la mauvaise résolution angulaire liée à la taille importante de l'échantillon et à la divergence du faisceau incident, ces pics ne sont pas forcément très localisés en $Q$. Cette diffusion cohérente complique l'analyse des données quasiélastiques puisqu'elle modifie le rapport entre les intensités élastique et quasiélastique. La solution consiste soit à analyser les zones angulaires ne présentant aucune contamination, soit à masquer leur position au niveau des analyseurs (à l'aide de bandes de cadmium). Dans tous les cas il est nécessaire de connaitre leur emplacement ainsi que leur évolution avec la température. La meilleure solution consiste à travailler avec un monocristal orienté de façon à eteindre un maximum de pics de diffraction.

Est ce que le signal quasiélastique est adapté à la fenêtre d'énergie?

Un problème important des spectromètres à rétrodiffusion est qu'il ne permettent l'analyse de la dynamique que sur une très petite fenêtre en énergie. Celle-ci n'est que $\sim 20$ fois supérieure à la résolution. Typiquement, des élargissements de $0.2 \mu \mathrm{eV}$ peuvent être observée. Cette limite dépend toutefois du système étudié et de l'intensité relative entre les pics élastique et quasiélastique. Lorsque les élargissements dépassent $\sim 5 \mu \mathrm{eV}$, les ailes (lorentziennes) sortent de la fenêtre en énergie. Un signal quasiélastique large peut ainsi facilement se confondre avec un bruit de fond et il convient de comparer les spectres aux enregistrements basses températures (où la diffusion devient purement élastique). Lorsque la largeur devient trop importante, il est necessaire de combiner les expériences, avec des mesures temps de vol (IN5, IN6), ou d'utiliser des variantes comme les spectromètres à monochromateurs chauffant (IN13, IN10b). Soulignons que l'incertitude sur la mesure des largeurs commence à devenir importante dès que celles ci dépassent $\Delta E_{\text {max }} / 3$.

\section{LES PROJETS D'INSTRUMENT}

\subsection{Comment améliorer le flux de neutrons incident sur l'échantillon ...}

Le problème principal associé aux spectromètres à $\mathrm{RD}$ est leur faible flux. Améliorer le flux sans trop dégrader la résolution spatiale, tel est le but des nouveaux spectromètres. Ces nouvelles techniques s'appuient sur l'utilisation de guides primaire de section importante, combinée avec une optique focalisante. La divergence au niveau du spectromètre primaire est augmentée et ne doit pas dépasser la résolution angulaire du spectromètre secondaire. Pour l'étude de la diffusion incohérente, la variation de l'intensité avec le vecteur de diffusion est généralement lente ce qui autorise une intégration angulaire sans conséquence importante. Paradoxalement, une augmentation de flux ouvre de nouvelles perspectives comme l'étude des phénomènes cohérents, extrèmement sensibles à la résolution angulaire. Aussi, il s'agit de trouver un bon compromis flux / résolution spatiale.

IN16 profite déjà d'un sytème de guides focalisants (focalisation verticale et horizontale) combiné à un monochromateur courbe (focalisation verticale). Un facteur 4 dans le flux a ainsi été obtenu par' rapport à IN10. L'optique focalisante semble être un alternative essentielle pour augmentater le flux de ces instruments. IN10 possède de même, un nez focalisant de petite taille qui permet de concentrer le faisceau sur des échantillons de petite dimension. 
Un projet actuellement à l'étude consiterait à déplacer IN16 en bout de guide et à améliorer la qualité des guides focalisants. Ceci devrait permettre de gagner un facteur 7 sur le flux actuel. Une autre alternative serait d'utiliser des neutrons ayant une longueur d'onde initiale plus éloignée que celle requise. L'élément optique permettant de "transformer" cette variation de longueur d'onde est constitué d'un cristal en mouvement parallèle à la surface de réflexion. Afin de rendre le système efficace, les cristaux doivent être montés sur un chopper et atteindre une vitesse de $250-300 \mathrm{~m} / \mathrm{s}$. Cette technique s'appelle la "phase space transformation" (PST). La transformation de cette distribution en longueur d'onde $(\delta \lambda / \lambda \sim 10 \%)$ conduit à une divergence plus importante du faisceau ( $\sim 15^{\circ}$ FWHM). L'utilisation de cette technique devrait permettre de gagner un facteur 4 dans le flux. Même si les gains estimés dans chacune de ces améliorations sont très certainement couplés, on peut raisonnablement envisager une augmentation importante du flux proche d'un facteur 10 .

\subsection{Comment améliorer la résolution spatiale}

La résolution spatiale des spectromètre à rétrodiffusion est déterminée par différents facteurs: la divergence du faisceau incident, la largeur de l'échantillon, le rayon des analyseurs, la dimension des cristaux, ainsi que la taille des détecteurs. Dans cette partie, nous ne nous intéresseront qu'à l'aspect détecteur.

Plusieurs configurations sont envisageables quant au réglage des analyseurs et des détecteurs. Dans la plupart des cas, les analyseurs sont directement focalisés sur les détecteurs. La résolution spatiale dépend alors de la largeur angulaire des analyseurs. Sur IN10 les détecteurs "ponctuels" font $5 \mathrm{~cm}$ de diamètre, et sont positionnés à $8 \mathrm{~cm}$ de l'échantillon. La résolution angulaire est proche de $26^{\circ}$. Une meilleure résolution peut être obtenue lorsque l'image des analyseurs est focalisée sur l'échantillon. Le système de détection est alors formé d'un ensemble de tubes, de longueur suffisante pour détecter la totalité de l'intensité diffusée. $\mathrm{La}$ résolution dépend alors du nombre de détecteurs couvrant le domaine accessible. Sur IN16, le multidétecteur comporte 20 tubes, pour une résolution de $6.5^{\circ}$. Le nombre de tube est augmenté sur IN13 (32) et donne un résolution de $5^{\circ}$. L'inconvenient des détecteurs multitubes tient au fait qu'il ne suivent pas les cones de Debye Scherrer ( $Q$ constante). De grandes modifications sont attendues aux faibles et aux grands angles de diffusion.

Lorsque la divergence du faisceau est faible, ce qui est le cas sur IN10, l'utlisation d'un multidétecteur bidimensionnel courbe (PSD) permet d'améliorer considérablement la résolution spatiale. Le détecteur, actuellement en période de tests, est de type ligne à retard. Celui ci présente une résolution de $2 \mathrm{~mm}$ à une distance de $25 \mathrm{~cm}$ de l'échantillon (résolution angulaire (horizontale et verticale) de $\sim 0.5^{\circ}$ ). Il devient alors possible de définir des masques suivant les cones de Debye Scherrer, et de regrouper les neutrons détectés dans chacun d'entre eux. Afín de profiter pleinement de cette très bonne résolution angulaire, un échantillon de forme cylindrique (et de diamètre $<1 \mathrm{~cm}$ ) doit être utilisé. Aussi, le besoin d'un flux important de neutrons est évident.

Il est possible avec un tel type de détecteur de localiser les pics de Bragg et de les exclure de l'analyse. Il est également envisageable de mesurer le diffractogramme de façon très précise en même temps que les spectres inélastiques. Cette option sera très bientot réalisable sur IN16 grâce à un ensemble de détecteurs installés aux pieds des analyseurs. Sur un spectromètre comme IRIS, la mesure de diffraction se fait depuis longtemps avec un nombre limité de détecteurs ponctuels. Ceci est effectivement possible avec un faisceau blanc et consiste en une analyse TdV du faisceau diffracté.

\subsection{La séparation de la diffusion cohérente et incohérente}

Bien que les détecteurs bidimensionnels courbes constituent une amélioration importante pour le traitement des pics de Bragg, la solution idéale nécessiterait l'utilisation de neutrons polarisés afin de séparer à la base, les contribution cohérente et incohérente. L'analyse de la polarisation entraînant diminution importante de l'intensité mesurée (facteur 4), elle nécessite un flux incident important. Une nouvelle version d'IRIS (appelée OSIRIS) [31] installée sur un nouveau guide est en cours de construction à ISIS. La séparation entre les contribution cohérente et incohérente est d'autant plus nécessaire dans les systèmes non-cristallins, comme certains polymères. Une autre application évidente est l'analyse de la diffusion magnétique. 


\section{Références}

[1] H. Maier-Leibnitz, Bayerische Akademie der Wissenschaften, Mathematisch-Naturwissenschaftliche Klasse, 16 (1966) 173

[2] B. Alefeld, Bayerische Akademie der Wissenschaften, Mathematisch-Naturwissenschaftliche Klasse, 11 (1966) 109

[3] A. Heidemann and B. Alefeld, "Review of backscattering techniques in neutron and X-ray scattering ", ILL Internal Report 91 HE 22 G (1992)

[4] C.G. Darwin, Phil. Mag. 27 (1914) 315 et 675

[5] B.Frick et al., Physica B 234-236(1997)1177

[6] http:/ww.ill.fr/YellowBook/IN5

[7] http://ww.ill.fr/YellowBook/IN11

[8] M.A. Neumann, D.F. Brougham, C.J. McGloin, M.R. Johnson, A.J. Horsewill and H.P.

Trommsdorff, J, Chem. Phys. 109 (1998) 7300

[9] D.F. Brougham, A.J. Horsewill et R.I. Jenkinson, Chem. Phys. Letts. 272 (1997) 69

[10] M. Birr, A. Heidemann et B. Alefeld, Nuc. Insts. and Methods 95 (1971) 435

[11] http://rrdjazz.nist.gov

[12] http://lansce.lanl.gov.lujan.instruments.htm

[13] http://www.ill.fr/YellowBook/IN10

[14] J.C. Cook, W. Petry, A. Heidemann et J-F Barthelemy, Nuc. Inst. Meths. In Phys. Res. A312 (1992) 553

[15] O.G. Randl et M.R. Johnson, Neutron News 8 (1997) 11

[16] http://www.ill.fr/YellowBook/IN13

[17] http://www.ill.fr/YellowBook/IN16

[18] B. Frick, A. Magerl, Y. Blanc et R.Rebesco, Physica B, 234-236 (1990) 1177

[19] http://www.isis.rl.ac.uk/CrystalAnalysers/iris.html

[20] B.Frick, L.J. Fetters, Macromolecules 27 (1994) 974

[21] B.Frick, D.Richter, SCIENCE 267 (1995)1939

[22] A. Tölle, H. Schober, J. Wuttke, O.G. Randl et F. Fujara, Phys. Rev. Lett. 80 (1998) 2374

[23] B. Frick, C. Alba-Simionesco, J. Hendricks et L. Willner; Prog. Theor. Phys.Suppl. 126 (1998)

[24] B. Frick et C. Alba-Simionesco. Physica B,1999, in press

[25] W. Press, "Single-particle rotations in molecular crystals", Springer Tracts in Modern Physics, vol.92 (1981)

[26] M. Prager et A. Heidemann, Chem. Rev. 97 (1997) 2933

[27] M.R. Johnson, M. Prager, H. Grimm, M.A. Neumann, G.J. Kearley et C.C. Wilson, Chem. Phys. 244 (1999) 49

[28] F. Fillaux et C.J. Carlile, Chem. Phys. Lett. 162 (1989) 188

[29] M.A. Neumann, M. Plazanet et M.R. Johnson, Phys. Rev. B (soumise jan 1999)

[30] K.H. Andersen, J. Bossy, J.C. Cook, O.G. Randl et J-L. Ragazzoni, Phys. Rev. Lett. 77 (1996) 4043 [31] http://www.isis.rl.ac.uk/CrystalAnalyseurs/osiris.html 